\section{Opioidverordnungen bei Arthrose}

van den Driest J] et al. Opioid prescriptions in patients with osteoarthritis: a population-based cohort study. Rheumatology 2020; 59:

2462-2470

Ärzte der Erasmus-Universität von Rotterdam untersuchten Inzidenz, Prävalenz und Trends von Opioidverordnungen sowie die langfristige Verschreibungsraten bei Arthrose-Patienten. Außerdem bewerteten sie die mit der Verschreibung von Opioiden verbundenen Patienteneigenschaften.

Mithilfe der IPCI (Integrated Primary Care Information)-Datenbank wurde eine bevölkerungsbasierte Kohortenstudie konzipiert. Die IPCI-Datenbank enthält elektronische Patientenakten von mehr als 1,5 Mio. niederländischen Patientinnen und Patienten. Inzidenz und Prävalenz von Opioidverordnungen von Arthrosepatienten älter als 30 Jahre, wurden für den Zeitraum 2008-2017 berechnet. Alle Opioid-Rezepte werden in den Niederlanden von Hausärzten oder Klinikärzten ausgestellt. Die Identifizierung erfolgte über den ATC-Code. Mit der logistischen Regressionsanalyse erfolgte die Beurteilung der Assoziationen von Patientenmerkmalen mit Opioidverordnungen.

Insgesamt wurden 157904 Patientinnen und Patienten mit Arthrose in die Studie eingeschlossen. Bei 56713 war eine Arthrose-Erstdiagnose erfolgt. 65,2\% der gesamten Patientengruppe war weiblich. An Begleiterkrankungen litten 55,2\% der Studienteilnehmer. Die häufigste Arthroseform war mit 27,8\% eine Gonarthrose. Bei $21,3 \%$ der Patientinnen und Patienten wurde eine Arthrose in mehr als einem Gelenk diagnostiziert. Zu Beginn der Nachbeobachtungsperiode zählte das Durchschnittsalter $66,6 \pm 12,5$ Jahre. Die mittlere Nachbeobachtungsdauer betrug 3 Jahre und 4 Monate. Im Verlauf der Nachbeobachtungszeit suchten $65 \%$ der Patientinnen und Patienten ihren Hausarzt mit einer anderen muskuloskelettalen Beschwerde neben der Arthrose auf.
In den letzten 10 Jahren blieb die Verschreibungsrate für Opioide insgesamt relativ stabil mit rund 100 inzidenten und 170 prävalenten Rezepte/1000 Personenjahre. Die Verschreibungsrate für starke Opioide stieg jedoch an, während die Verschreibungsrate für schwache Opioide abnahm. So kletterte die inzidente Verschreibungsrate für Oxycodon von 7,1/1000 Personjahre auf 40,7/1000 Personenjahre und für Fentanyl von 4,2 auf 7,4/1000 Personenjahre. Die Verschreibungsrate für Paracetamol/Codein verringerte sich hingegen von 63,0 auf 13,3/1000 Personenjahre. Pro Follow-upJahr wurde bei $3 \%$ der Patienten mit inzidenter Arthrose eine Langzeitanwendung festgestellt.

Die logistische Regressionsanalyse ergab, dass zunehmendes Alter, eine Arthrose in 2 oder mehr Gelenken sowie zusätzliche muskuloskelettale Beschwerden mit vermehrten Opioidverschreibungen assoziiert waren. Männer wurden seltener Opioide als Frauen verschrieben.

\section{FAZIT}

Die Verschreibungsraten für Opioide blieben stabil, allerdings nahmen die Verschreibungen für stark wirksame Opioide zu, während die für schwache Opioide abnahmen. Da der Nutzen von Opioiden bei Arthrose-Schmerzen fraglich ist und Nebenwirkungen häufig sind, so das Autorenteam, sollten Opioide mit Vorsicht verschrieben werden.

Richard Kessing, Zeiskam 